\title{
Putting genetic variants to a fitness test
}

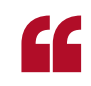

high-throughput genome-editing approaches to test the functionality of genetic variants

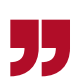

Enormous strides have been made in linking genetic variation and complex traits, with genome-wide association studies reporting hundreds or even thousands of associated variants. However, functional testing of these variants has lagged behind and is mostly done in low-throughput set-ups. Two studies in Nature and Cell now report the application of high-throughput genome-editing approaches to test the functionality of genetic variants in human and yeast cells, respectively, by assessing their effects on cellular fitness.

Several variants in the gene $B R C A 1$ are associated with an increased risk of breast and ovarian cancer. Yet, most single-nucleotide variants (SNVs) in $B R C A 1$ are classified as variants of unknown significance (VUS); it is currently unclear whether they affect protein function or health. To experimentally test all BRCA1 variants, Findlay et al. performed saturation genome editing (SGE) in the near-haploid cancer cell line HAP1. Genes in the homologydirected DNA repair (HDR) pathway, including BRCA1, are essential for the survival of HAP1 cells. Hence, if a genetic variant disrupts BRCA1 activity, this effect would be reflected in reduced cellular viability.

The authors targeted the 13 exons of $B R C A 1$ that encompass the RING and BRCT domains and designed Cas9 guide RNA (gRNA) constructs

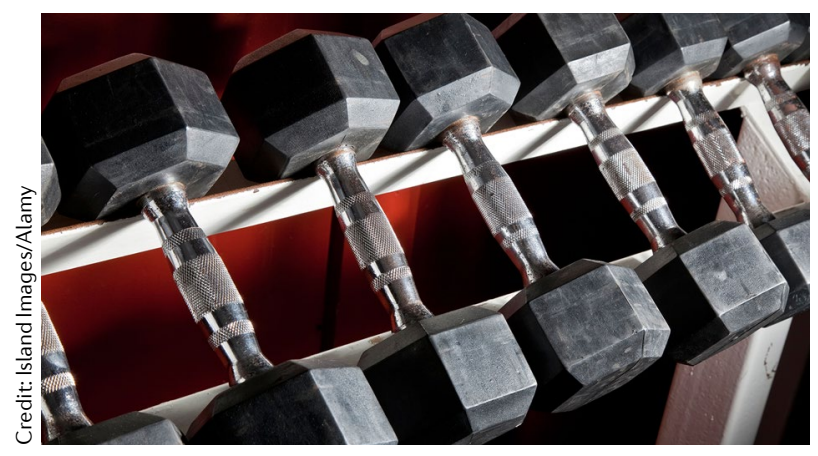

and SNV libraries of repair templates (including each possible SNV in an exon within approximately $100 \mathrm{bp}$ of the genomic sequence). At day 5 and 11 after transfection, cells were assayed by targeted gDNA and RNA sequencing to determine SNV abundancies and, thus, cell numbers. Thereby, function scores for 3,893 BRCA1 SNVs were obtained, $72.5 \%$ of which were classified as functional, $21.1 \%$ as nonfunctional and $6.4 \%$ as intermediate.

The results showed good agreement with known variants, present in ClinVar, a database of disease-relevant human genetic variants. Of note, $25 \%$ of VUS and $49 \%$ of variants with conflicting previous reports were flagged as nonfunctional. The authors estimate that their approach has $>95 \%$ accuracy in predicting the functional outcome of a genetic variant in BRCA1 and stress the importance of their findings for the interpretation of clinical sequencing results.

The work by Findlay et al. relied on the co-transfection of separate high-copy number plasmids encoding the Cas9 gRNA and repair templates, requiring individual experimental set-ups for each exon for efficient pairing of gRNA and repair templates. By contrast, a new approach for massively parallel genome editing developed by Sharon, Chen and Khosla et al. makes use of retrons that can be engineered to carry both elements on the same molecule.

Retrons are bacterial retroelements that encode a reverse transcriptase gene and a single-stranded DNA (ssDNA)-RNA hybrid. In this method, called Cas9 retron precise parallel editing via homology (CRISPEY), the Escherichia coli Ec86 retron was fused to single gRNA sequences, and parts of the retron sequence were replaced with a 100 nucleotide donor sequence for HDR. A total of 16,006 SNVs and insertions and deletions of the vineyard yeast isolate RM were introduced into the lab strain BY (which expresses Cas9) using 32,000 gRNA-donor pairs (in a single set-up). The fitness effects were measured by sequencing to determine the abundance of each strain following growth over 25 generations.

In total, 171 variants and 17 variants affected fitness more than $1 \%$ and $5 \%$, respectively, per generation. These variants were enriched in cis-regulatory elements, specifically in promoter regions and transcription factor binding sites, which is in line with the general assumption that trait-associated variants affect largely non-coding parts of the genome.

CRISPEY has multiple advantages that increase editing efficiencies: it does not require the use of highcopy number plasmids; single gRNAs and the HDR template are encoded on the same molecule; and the generated donor template is ssDNA. Because of the retrons' versatility and functionality, not just in yeast but also in mammalian cells, the authors anticipate that CRISPEY can be adapted to answer questions about genetic associations in higher organisms. Moreover, CRISPEY could be adapted to measure outcomes beyond cellular fitness if coupled to a fluorescent reporter gene, for example.

Taken together, the two studies highlight the utility of highthroughput genome editing for the functional analysis of multiple genetic variants.

Michelle Trenkmann, Associate Editor, Nature Communications

ORIGINAL ARTICLES Findlay, G. M. et al. Accurate classification of BRCA1 variants with saturation genome editing. Nature https://doi.org/10.1038/ s41586-018-0461-z (2018)| Sharon, E., Chen, S.-A.A. \& Khosla, N. M. et al. Functional genetic variants revealed by massively parallel precise genome editing. Cell https://doi.org/10.1016/j.cell.2018.08.057 (2018) 